\title{
Contribuciones y retos de la economía de la salud en Cuba
}

\author{
Contributions and challenges of health economics in Cuba
}

\author{
Dr. C. Ana María Gálvez González \\ Escuela Nacional de Salud Pública. La Habana, Cuba.
}

\begin{abstract}
RESUMEN
El objetivo de este trabajo es realizar una exposición crítica sobre el desarrollo que ha tenido la economía de la salud en el contexto sanitario cubano. El mismo se ha producido en tres ámbitos en los que esta disciplina ha desplegado sus actividades: la docencia, la investigación y la participación en la toma de decisiones. Luego de más de treinta años de la aparición esta disciplina en Cuba, es posible señalar un conjunto de aciertos y retos. El área más fortalecida es la docencia, que aún con las dificultades que pueda presentar, es la fuente básica que promueve la investigación en este campo. El problema más difícil es la aplicación de los resultados de las investigaciones a la toma de decisiones y el fortalecimiento de la introducción de los conocimientos teóricos al quehacer diario. El desarrollo de la economía de la salud en Cuba presenta similitudes y diferencias respecto al experimentado por otros contextos. Este comportamiento puede asociarse a particularidades específicas del contexto sanitario cubano y de las características del modelo económico que desarrolla el país. En Cuba, en los próximos años, la integración docencia, investigación y toma de decisiones en economía de la salud ha de acentuarse, esta necesidad se convierte en un punto de partida. Es la mejor forma para conocer los resortes económicos de nuestra realidad en el campo sanitario y de mantener una relación provechosa con lo más avanzado del pensamiento económico.
\end{abstract}

Palabras clave: economía de la salud, docencia, investigación, toma de decisiones.

\section{ABSTRACT}

The objective of this paper was to submit a critical presentation of the development of health economics in the Cuban health context. Health economics in Cuba has been developed in three main areas: education, research and participation in decision making. After more than thirty years of the emergence of this discipline in Cuba, it is 
possible to mention a group of strengths and challenges. Education is the area with the greatest improvements; despite some difficulties that may exist, it is the basic source of research promotion in this field. The biggest challenges are to incorporate the research results into the decision making process and to reinforce the introduction of the theoretical knowledge to the daily work. The development of health economics in Cuba presents some similarities and differences if compared to that of other settings. This behavior may be associated to specific characteristics of the Cuban health system and the characteristics of the economic model that the country puts into practice. In the forthcoming years, the integration of education, research and decision making in the field of health economics needs to be reinforced, since it is a starting point and the best way to know the key economic elements of our reality in the health care area and to keep an advantageous relationship with the most advanced economic thinking.

Key words: health economics, education, research, decision making.

\section{NTRODUCCI ÓN}

En los últimos años, el país se ha venido enfrentando a una ardua labor, dirigida a la actualización del modelo económico cubano. El VI Congreso del Partido Comunista de Cuba (PCC), ${ }^{1}$ y, posteriormente, su I Conferencia ${ }^{2}$ han definido los principios y los objetivos básicos en los que debe basarse esta tarea. Este proceso se produce en medio de una grave crisis de la economía mundial, que en el caso cubano se agrava como consecuencia de más de 50 años de bloqueo y agresiones económicas. El Sistema Nacional de Salud (SNS) participa en el mismo en condiciones particulares. Luego de una larga historia de priorización de la salud pública, se han alcanzado importantes resultados que colocan a Cuba entre los países más avanzados en este campo. De aquí, que mantener y mejorar los logros alcanzados se convierte en un objetivo que reclama un trabajo intenso y decisiones cuidadosas. Desde el año 2010 se cuenta con un documento que orienta las transformaciones necesarias ${ }^{3}$ encaminadas a perfeccionar el sector y dar respuesta a los reclamos de elevación de la eficiencia y de aprovechamiento de los escasos recursos disponibles, a los que ha convocado la máxima dirección del país. En este contexto, la economía de la salud tiene un importante papel que jugar, acompañando a otras disciplinas científicas que conforman la salud pública cubana.

Encontrar un espacio en el ámbito de la salud pública no ha sido un quehacer sencillo para la economía de la salud. El mismo, que se ha abordado con dedicación creciente desde hace más de 30 años, ha experimentado en los tiempos más recientes una mayor sensibilidad dentro del sector sanitario en particular $y$, en general, en todos los espacios donde se tomen decisiones que afecten la salud de la población. La toma de conciencia sobre la importancia del análisis del presupuesto y su aplicación a través de los consejos económicos en las instituciones de salud, el análisis del volumen y composición del gasto sanitario, el perfeccionamiento en las estructuras de dirección de las instituciones de salud y una mayor preocupación por la sistematización de la evaluación económica como instrumento para la toma de decisiones, constituyen ejemplos que ilustran como conceptos, métodos e instrumentos propios de la Economía de la Salud han sido capaces de aportes apreciables al desarrollo de la salud. 
El desarrollo de la economía de la salud en el contexto sanitario se ha producido en tres ámbitos en los que esta disciplina ha desplegado sus actividades: la docencia, la investigación y la participación en la toma de decisiones.

Mediante los procesos docentes se forma a los especialistas en economía de la salud y se dota a otros profesionales de habilidades en esta disciplina. Al mismo tiempo se desarrollan procesos investigativos que se integran en la docencia y aportan, directa o indirectamente, elementos que contribuyen a enfrentar la toma de decisiones. Este último aspecto, seguramente el más complejo de los tres, se nutre de resultados de la docencia y la investigación, a la vez que aporta principios y contenidos que deben ser incluidos en aquellos campos. Se trata de una interrelación compleja y, hasta cierto punto ideal que, de funcionar de manera coordinada y dirigida hacia un mismo fin puede tener una influencia decisiva en el desarrollo de la salud pública cubana.

El objetivo de este trabajo es realizar una exposición crítica sobre el desarrollo que ha tenido la economía de la salud en el contexto sanitario cubano.

\section{DOCENCI A E I NVESTI GACI ÓN EN LOS I NI CI OS DEL DESARROLLO DE LA ECONOMÍ A DE LA SALUD EN CUBA}

La inclinación por los temas económicos asociados a la salud tiene en Cuba raíces históricas profundas. En la vida y obra de pensadores cubanos del campo sanitario se puede apreciar el interés y la preocupación por causas y efectos económicos de la enfermedad y la política sanitaria. ${ }^{4-7}$ De la misma forma, se pueden encontrar interesantes aportes sobre salud y servicios sociales entre exponentes del pensamiento político y económico cubano. ${ }^{8-11}$

A principio de los 70 del siglo pasado se constatan las primeras evidencias de formación de salubristas cubanos en temas económicos aplicados a la salud. Los mismos se insertaban en cursos de economía que impartían la Universidad de La Habana y otras instituciones nacionales de perfil económico. Durante esta etapa existía un fuerte intercambio con instituciones de salud extranjeras, básicamente del campo socialista, donde los temas económicos de la salud se introdujeron desde la perspectiva de la salud pública y de la higiene social. ${ }^{12,13}$

A fines de esa década, se inicia la experiencia de impartir docencia en temas económicos dentro del sector de la salud cubano, con el diseño e impartición de cursos cortos de temas económicos en el Instituto de Desarrollo de la Salud (IDS). En los 80 se consolidan estos temas con cursos de planificación y contabilidad dentro de la formación de la especialidad de Administración de Salud y elementos de la economía como determinante en el estado de salud de la población en el Curso Internacional de Salud Pública.

En esta etapa se producen las primeras tesis relacionadas con el tema. Se dan así los primeros pasos en una práctica que va a caracterizar el desarrollo de la investigación en temas vinculados a la economía. La docencia empieza a jugar un papel determinante en este campo, el cual se hará cada vez más evidente con el paso de los años.

En los 90 se produce en América Latina un proceso de reforma sanitaria en los sistemas de salud. Estas reformas se habían propuesto, en principio, la introducción de criterios de eficiencia en la forma en que se organizaba la provisión de los servicios de salud. Se promueve entonces la economía de la salud como una herramienta valiosa para el diseño de los sistemas de salud, así como para la comprensión del 
funcionamiento de los mercados de salud y de las principales dificultades en la puesta en marcha de las reformas. En esta etapa se crea la Red de Economía y Financiamiento de la Salud (REDEFS), promovida por la Organización Panamericana de la Salud. Esta red significó un fuerte impulso a la economía de la salud en los países integrantes y el fortalecimiento del intercambio en este tema entre países latinoamericanos.

La inserción de Cuba en la REDEFS, permitió aprovechar los espacios de formación en economía de la salud que brindaba la misma y facilitó el intercambio de investigaciones entre centros. Los encuentros de REDEFS se convirtieron en una tribuna interesante para mostrar los logros alcanzados por la salud pública cubana así como las experiencias en términos de economía y financiamiento de la salud desde la perspectiva social y gratuita. Fue un espacio importante para enfrentar y discutir las políticas neoliberales sobre la salud, tema imperante en esa etapa. Cuba también ganó en experiencias de integración a la región y en la formación de docentes cubanos dedicados a la especialidad de economía de la salud.

En 1997, en la Facultad de Salud Pública (FSP) se inicia el diplomado de economía de la salud. La presencia de esta figura académica consolida la comunidad académica cubana en economía de la salud. Esta experiencia se extendió a todo el país, lo que impulsó el desarrollo de otros polos científicos en economía de la salud. El más importante de ellos se ubicó en la provincia de Santiago de Cuba, donde además se trabajaba en otras experiencias interesantes en este campo, como la celebración sistemática de simposios de economía de la salud, que estimularon la realización de estudios sobre este tema. En 1998, se aprueba por Resolución 61/98 del Ministerio de Educación Superior (MES) un programa de Maestría en Economía de la Salud, en esta provincia.

Es necesario destacar que, entre 1997 y 1999, un amplio equipo interdisciplinario, que incluyó a los alumnos y profesores del Diplomado en Economía de la Salud intervino en una importante investigación internacional sobre evaluación económica de la atención prenatal que parte de una investigación más amplia sobre atención prenatal, financiada por la OMS. Otros estudios multicéntricos de alcance internacional como el proyecto para el desarrollo de la evaluación económica en América Latina (NEVALAT) ${ }^{14}$ promovieron hacia un salto cualitativo en la investigación de economía de la salud en Cuba.

En el año 2001 se da un proceso de perfeccionamiento de la maestría, con participación de la Escuela Nacional de Salud Pública (ENSAP), a partir del cual se empieza a brindar esta figura académica en dos sedes, La Habana y Santiago de Cuba. Desde el año 2008, la sede de la ENSAP cumple con la condición de excelencia según las normas del patrón de calidad del MES. ${ }^{15}$ Hasta el año 2011, ha tenido cuatro ediciones, la tercera de las cuales, se brindó a los colaboradores cubanos en la República Bolivariana de Venezuela. Adicionalmente, la disciplina ha sido incluida en otras maestrías, así como en cursos cortos y diplomados.

La investigación ha sido el eje conductor en la maestría y, más recientemente, en la formación de doctores. De aquí que se haya producido una creciente producción científica a partir de tesis de maestría y doctorado. ${ }^{16}$ Una parte importante de estas últimas ha obtenido premios de alto nivel otorgados por el MINSAP o el MES. Se ha podido apreciar en los últimos cinco años un crecimiento en el número de publicaciones en revistas científicas, libros y otros tipos de publicaciones. La celebración de eventos científicos sistemáticos de economía de la salud así como la presencia de trabajos del tema en otros espacios científicos, constituyen elementos alentadores en cuanto a la marcha de la disciplina. 


\section{EL ROL DE LA ECONOMÍ A DE LA SALUD EN LA TOMA DE DECISIONES EN EL CONTEXTO SANITARIO CUBANO}

Respecto a la relación economía de la salud y toma de decisiones se puede afirmar que el SNS ha atravesado diferentes etapas. Han existido momentos de estrecha relación y de desarrollo de trabajos conjuntos de gran éxito para el sistema y también periodos de separación y trabajo aislado con sus consecuencias negativas. Lo cierto es, que se aprecia un creciente interés en el uso de las herramientas económicas tanto desde la dirección del sector de la salud como desde el primer nivel de dirección del país.

La economía de la salud es una disciplina cuyos resultados deben estar fuertemente vinculados a la práctica cotidiana. Esta disciplina se asocia a trabajos de carácter empírico que deben servir para evaluar antes o después las medidas de política sanitaria y económica. De ahí su estrecha relación con la toma de decisiones.

Existen evidencias en el país de estudios de economía de la salud que han contribuido a la toma de decisiones. ${ }^{17-19}$ Se puede apreciar un incremento en la preocupación de los encargados de la toma de decisiones por incluir el tema económico en su quehacer diario. Otro elemento de interés es la motivación con la estandarización de instrumentos de evaluación económica en aras de ganar transparencia y transferabilidad en las evaluaciones económicas que se realizan. ${ }^{20-22}$

Un elemento que ha tomado fuerza de análisis es la valoración de las implicaciones económicas de las decisiones que se toman en salud en relación con otros sectores de la economía nacional. De la misma forma que las decisiones que en otros sectores se adopten, tienen repercusiones en la salud de la población.

Ya van quedando atrás los tiempos en los que algunos directivos de salud pensaran que los problemas económicos y del control interno ${ }^{23}$ en una institución eran inherentes solamente a los economistas y contadores, o al equipo de dirección. Lejos también ha quedado la idea de confundir a la economía con la contabilidad, o pensar que la economía solamente trata del dinero. Esta toma de conciencia es resultado en parte de la superación sistemática que reciben los cuadros y directivos en temas de economía así como de la prioridad que el país asigna en estos momentos a la formación de sus cuadros en temas económicos como parte de la actualización del modelo económico cubano.

\section{ACIERTOS Y RETOS EN EL DESARROLLO DE LA ECONOMÍ A DE LA SALUD EN CUBA}

El área más fortalecida es la docencia que aún con las dificultades que pueda presentar, es la fuente básica que promueve la investigación. El problema más difícil es la aplicación de los resultados de las investigaciones a la toma de decisiones y el fortalecimiento de la introducción de los conocimientos teóricos al quehacer diario, aspecto este que en ocasiones ha sido suplantado por el empirismo o la inmediatez de la cotidianeidad.

Luego de más de 30 años de la aparición de la economía de la salud en Cuba, es posible señalar los principales aciertos de este proceso. En primer lugar, se ha alcanzado estabilidad y calidad en el sistema de cursos que se imparten, existe voluntad política para el desarrollo de la disciplina, el país cuenta con dos polos de formación docente y con un claustro de profesores de alto nivel científico y experiencia práctica, se han incorporado las tecnologías de la información y las 
comunicaciones en la formación, se han diseñado bases de datos de tesis, encuentros virtuales de estudiantes y profesores, foros de discusión, entre otros. El prestigio alcanzado en la formación en economía de la salud en Cuba ha fomentado la demanda de cursos y profesores cubanos en otros países, fundamentalmente en países latinoamericanos que buscan la experiencia cubana como un modelo alternativo a las formas de planificación y asignación de recursos presentes en sus realidades.

No obstante se identifica un conjunto de retos que deben ser enfrentados:

- Identificar líneas de investigación prioritarias en economía de la salud que contribuyan al desarrollo eficiente de la salud pública cubana.

- Fomentar estudios multicéntricos y otros tipos de colaboración que potencien los resultados y permitan un uso eficiente de los recursos humanos formados en esta disciplina.

- Estimular la incorporación de profesores e investigadores jóvenes dedicados a tiempo completo a la disciplina.

- Incrementar la docencia y la investigación en economía de la salud en otras provincias además de La Habana y Santiago de Cuba, así como sistematizar el intercambio entre centros de formación.

- Extender la formación en economía de la salud hacia el pregrado tanto en la formación en las carreras de medicina y estomatología como en las facultades de economía.

- Estimular la dedicación al análisis de la dimensión teórica de la disciplina e invitar a economistas de otros sectores a participar en el debate teórico metodológico de los problemas de salud.

- Diseñar y validar sistemas de información necesarios para las investigaciones económicas en salud, por ejemplo, los sistemas de costos aun deficientes en gran parte de las instituciones del SNS.

- Disponer de un espacio para la publicación sistemática de los resultados en el campo de la economía de la salud.

Al comparar las contribuciones y los retos planteados en este trabajo con análisis similares realizados en otros contextos, algunos de ellos con más años recorridos en el tema y mayor desarrollo económico, ${ }^{24-31}$ se pueden apreciar similitudes. Por ejemplo; la escasez de profesionales dedicados a tiempo completo a la economía de la salud, la lentitud en el proceso de maduración de una comunidad científica en el tema, pocos recursos y financiamientos intermitentes destinados a la investigación, limitaciones en la coordinación con las necesidades en política sanitaria, incongruencias metodológicas (sobre todo en el área de evaluación económica) y contacto no sistemático con expertos de otros países.

Existen también diferencias con esos mismos contextos, algunas de ellas favorables en el caso cubano, entre las que se pueden mencionar la manifiesta voluntad política para el desarrollo de la disciplina, el diálogo interdisciplinario con epidemiólogos, bioestadísticos y farmacéuticos y las bondades del carácter gratuito de la educación cubana para la formación de profesores e investigadores, fortalezas que no están siendo aprovechadas en su máximo potencial. Otra diferencia favorable en el es la 
percepción de la salud como un logro social, se hace énfasis en los análisis en factores determinantes del estado de salud de la población más allá del enfoque clínico.

En los próximos años la integración docencia, investigación y práctica en economía de la salud en Cuba ha de acentuarse, esta necesidad se convierte en un punto de partida. Es la mejor forma para conocer los resortes económicos en nuestro contexto sanitario y de mantener una relación provechosa con lo más avanzado del pensamiento económico. El éxito de la economía de la salud será juzgado por la medida en que mejore la toma de decisiones.

\section{Agradecimientos}

A Manuel Álvarez Muñiz por sus consideraciones.

\section{REFERENCI AS BI BLI OGRÁFICAS}

1. Partido Comunista de Cuba. Lineamientos de la política económica y social del Partido y la Revolución. Abril de 2011.

2. Partido Comunista de Cuba. Resolución de la primera conferencia nacional acerca de los objetivos de trabajo del partido. Diario Granma [Internet]. [citado 30 Ene 2012]. Disponible en: http://www.cubadebate.cu/especiales/2012/01/30/resolucionde-la-primera-conferencia-nacional-acerca-de-los-objetivos-de-trabajo-del-partido/

3. Ministerio de Salud Pública. Transformaciones necesarias en el sistema de salud pública [Internet]. 2010 [citado 30 Ene 2012]. Disponible en:

http:/files.sld.cu/editorhome/files/2010/11/transformaciones-necesarias-saludpublica.pdf

4. Rojas Ochoa F. Políticas sociales y salud de la población. Rev Cubana Salud Pública [Internet]. 2011 [citado 4 Jun 2012 ];37(4):362-5. Disponible en:

http://scielo.sld.cu/scielo.php?script=sci arttext\&pid=S0864$\underline{34662011000400001 \& \operatorname{lng}=\mathrm{es}}$

5. Rojas Ochoa F. Pobreza y Salud. Rev Cubana Salud Pública [Internet]. 2007 [citado 4 Jun 2012 ];33(4).I Disponible en:

http://scielo.sld.cu/scielo.php?script=sci arttext\&pid=S086434662007000400001\&lng=es

6. Ramírez Márquez A, López Pardo CM. A propósito de un sistema de monitoreo de la equidad en salud en Cuba. Rev Cubana Salud Pública [Internet]. 2005 [citado 4 Jun 2012];31(2). Disponible en:

http://scielo.sld.cu/scielo.php?script=sci arttext\&pid=S0864$\underline{34662005000200002 \& \operatorname{lng}=\mathrm{es}}$ 
7. Delgado García G. Doctor Gustavo Aldereguía Lima: luchador e higienista social. Rev Cubana Salud Pública [Internet]. 2012 [citado 4 Jun 2012]; 38(2):183-91. Disponible en: http://scielo.sld.cu/scielo.php?script=sci arttext\&pid=S0864 $\underline{34662012000200002 \& \operatorname{lng}=\mathrm{es}}$

8. Castro Ruz F. La historia me absolverá. Texto completo [Internet]. 2012 [citado 4 Jun 2012]. Disponible en: http://www.granma.cubaweb.cu/marti-moncada/jm01.html

9. CEPAL/INIE/PNUD. Política social y reformas estructurales en Cuba a principios del siglo XX1. LC/MEX/GI [Internet]. 2004 [citado 4 Jun 2012]. Disponible en: http://www.eclac.org/publicaciones/xml/3/15003/L2091-01.pdf

10. Everleny O. Economía y Bienestar en Cuba. Una agenda a completar. Centro de Estudios de la Economía Cubana [Internet]. Universidad de La Habana: Centro; 2007 [citado 4 Jun 2012]. Disponible en:

http://focal.ca/pdf/cuba Everleny\%20Perez\%20Villanueva economia\%20bienestar\% 20Cuba September\%204\%202007 Montreal.pdf

11. González A. Economía y Sociedad: los retos del modelo económico. Rev Cuba: Investigación Económica. 1997;(3-4):4.

12. Zhilinskas Youzas Yu. Metodología de Planificación y Financiamiento de la salud Pública Soviética y sus Aspectos Económicos. La Habana: Ministerio de Salud Pública; 1979.

13. Serenko A F. Ermakov VV. Higiene Social y organización de la salud pública. Moscú: Editorial Mir; 1984.

14. Iglesias CP, Drummond MF, Rovira J. Nevalat Project Group. Health Care Decision Makinng processes in Latin America: problems and prospects for the use of economic evaluation. Internat J Technol Assesment. 2005:21(1):1-14.

15. Louro Bernal Isabel, Perdomo VI, Gálvez González AM, Sanabria Ramos G.

Estrategia de preparación para la evaluación externa de maestrías. Educ Med Super [Internet]. 2011 [citado 5 Jun 2012];25(2):107-15. Disponible en:

http://scielo.sld.cu/scielo.php?script=sci arttext\&pid=S0864$\underline{21412011000200008 \& \operatorname{lng}=e s}$

16. Gálvez González AM, García Fariñas A, Portuondo Sánchez C. Producción científica en la maestría de economía de la salud desde la perspectiva de las tesis. Educ Med Super [Internet]. 2011 [citado 5 Jun 2012];25(4):389-97. Disponible en: http://scielo.sld.cu/scielo.php?script=sci arttext\&pid=S0864 $\underline{21412011000400001 \& \operatorname{lng}=\text { es }}$

17. Collazo Herrera M, Gálvez AM, García A, Lara C. La farmacoeconomía en Cuba. Implementación de su aplicación y proyecciones de trabajo. Rev Española Economía Salud. 2010;9(2):53-8.

http://scielo.sld.cu 
18. García Fariñas A. Empleo del Análisis envolvente de datos para la medición de la eficiencia de instituciones del Sistema Nacional de Salud. Algunas experiencias en Cuba. Investigación aplicada a la salud. Una mirada desde la Investigación de Operaciones. La Habana: Universidad de La Habana; 2010.

19. Lara Bastanzuri C, Calvo Barbados DM, Pérez Peña J. Generalidades del Cuadro Básico de Medicamentos en Cuba. Modificaciones en el 2010 que determinan cambios en el abordaje terapéutico. Rev Cubana Farm [Internet]. 2010 [citado 5 Jun 2012];44(3):283-6. Disponible en: http://scielo.sld.cu/scielo.php?script=sci arttext\&pid=S003475152010000300001\&lng=es

20. Gálvez González AM. Guía metodológica para la evaluación económica en salud: Cuba, 2003. Rev Cubana Salud Pública [Internet]. 2004 [citado 4 Jun 2012 ];30(1). Disponible en: http://scielo.sld.cu/scielo.php?script=sci arttext\&pid=S0864 34662004000100005\&lng=es

21. García Fariñas A, Gálvez González AM, García Rodríguez JF. Aspectos metodológicos críticos en las evaluaciones económicas de salud en el contexto cubano. Rev Cubana Salud Pública [Internet]. 2010 [citado 4 Jun 2012];36(3):233-5. Disponible en: http://scielo.sld.cu/scielo.php?script=sci arttext\&pid=S0864 34662010000300007\&lng=es

22. Gálvez González AM, Garcia Fariñas A, Portuondo Sánchez C, Lara Bastanzuri C, Collazo Herrera M. Evaluación económica en salud y toma de decisiones en el contexto sanitario cubano. Rev Cubana Salud Pública [Internet]. 2012 [citado 4 Jun 2012];38(2):253-62. Disponible en:

http://scielo.sld.cu/scielo.php?script=sci arttext\&pid=S0864$\underline{34662012000200008 \& \operatorname{lng}=\mathrm{es}}$

23. Contraloría General de la República. Revista Auditoría y control. Resolución No. 60/11. 2011(24):34-43.

24. García Altés A. Twenty years of health care economic analysis in Spain: are we doing well? Avances en la gestión sanitaria: implicaciones para la política, las organizaciones sanitarias y la práctica clínica. Palma de Mallorca: Asociación de Economía de la Salud; 2000.

25. Eddama O, Coast J. Use of economic evaluation in local health care decisionmaking in England: a qualitative investigation. Health Policy. 2009;89(3):261-70.

26. Yothasamut J, Tantivess S, Teerawattananon Y. Using economic evaluation in policy decision making in Asian countries: mission impossible or mission probable? Value Health. 2009;12(suppl 3):S26-30.

27. Restrepo Zea Jairo H. Introducción a la economía de la salud en Colombia. Centro de Investigaciones y Consultorías. Antioquia: Universidad de Antioquia, Facultad de Ciencias Médicas; 2008.

http://scielo.sld.cu 
28. Drummond M. Twenty years of using economic evaluations for reimbursement decisions. What have we achieved? York: University of York, Centre for Health Economics; 2012.

29. Drummond M, Botten G, Hakkinen U, Pedersen K. An Evaluation of Swedish Health Economics Research [folleto]. Stockholm: Swedish Council for working life and social research; 2006.

30. Gurgel de Andrade EI. Pesquisa e produção científica em economia da saúde no Brasil. Rev Adm Pública [Internet]. 2007 [cited 2010 Oct 27];41(2):211-35. Available from: http://www.scielo.br/scielo.php?script=sci arttext\&pid=\$0034 $\underline{76122007000200003 \& \operatorname{lng}=e n \& n r m=i s o \& t \operatorname{lng}=p t}$

31. Arredondo A. Temas selectos en sistemas de salud: costos,financiamiento, equidad y gobernanza. Conceptos, tendencias y evidencias. 2da ed. México, D.F.: Universidad Autónoma de Yucatán, Facultad de Ciencias Antropológicas; 2009.

Ana María Gálvez González. Escuela Nacional de Salud Pública. Calle 100 No. 1132 e/ E y Perla. Altahabana, municipio Boyeros. La Habana, Cuba.

Correo electrónico: galveza@infomed.sld.cu 